\title{
El bienestar subjetivo en la adolescencia: Estudio comparativo de dos Comunidades Autónomas en España
}

\author{
Ferran Casas $^{2 *}$, Sergio Fernández-Artamendi², Carme Montserrat ${ }^{1}$, Amaia Bravo ${ }^{2}$, Irma Bertrán ${ }^{1}$ y Jorge F. del Valle ${ }^{2}$ \\ ${ }^{1}$ ERIDIQV. Instituto de Investigaciones sobre Calidad de Vida (IRQV), Universitat de Girona. bttp:/ / wmw.udg.edu/eridiqv \\ ${ }^{2}$ Grupo de Investigación en Familia e Infancia (GIFI) Universidad de Oviedo. www.gifi.es
}

\begin{abstract}
Resumen: Se analiza la comparabilidad interlingüística e intercultural del bienestar subjetivo durante la adolescencia, a partir de dos muestras de Asturias y de Cataluña de entre 15 y 18 años. Se utilizan como indicadores psicosociales de bienestar tres escalas: La SWLS de Diener, Emmons, Larsen y Smith (1985), escala libre de contexto (context free), el PWI de Cummins, Eckersley, van Pallant, Vugt y Misajon (2003), que evalúa el bienestar personal por ámbitos, y una escala de ítem único sobre satisfacción global con la vida (Overall Life Satisfaction $=$ OLS).

Utilizando modelos de Análisis Factorial Confirmatorio, el PWI muestra un buen ajuste para ambas muestras, avalando la comparabilidad de los resultados entre ambas poblaciones. Sus puntuaciones globales resultan más altas entre los adolescentes asturianos que entre los catalanes, igual que la OLS.

La SWLS muestra un mal ajuste con los términos constantes restringidos, que añadido al hecho que el alpha de Cronbach aumentaría si el ítem 5 fuera suprimido, abunda en la idea que esta escala funciona de manera distinta en contextos socio-culturales diferentes y mantiene abiertas las dudas sobre si es apropiada su utilización como instrumento comparativo entre poblaciones de características lingüísticas y/o socioculturales distintas.

Palabras clave: Bienestar subjetivo; adolescencia; PWI; SWLS; satisfacción vital; bienestar personal; análisis factorial confirmatorio; modelos de ecuaciones estructurales.
\end{abstract}

\section{Introducción}

Según Huebner (2004), los estudios sobre el bienestar subjetivo de poblaciones adolescentes son muy escasos en comparación con los realizados con adultos. Apenas existen investigaciones que comparen el bienestar de los adolescentes de contextos socio-culturales distintos y los que lo han hecho, en general, han utilizado un único instrumento de entre los pocos disponibles para uso con poblaciones no clínicas.

En la comparación intercultural del bienestar infantil y adolescente parece que una publicación de UNICEF, la Report Card 7 (Adamson, 2007), ha establecido una especie de punto y aparte en el debate internacional. En primer lugar, al defender que un sistema de indicadores para evaluar el bienestar infantil también requiere la inclusión de indicadores de bienestar subjetivo, igual que en poblaciones adultas; y en segundo lugar por señalar que tan sólo se han encontrado datos publicados de un mínimo número de países utilizando la escalera de Cantril (1965).

Obviamente, la utilización de sólo una escala de ítem único para comparar el bienestar subjetivo infantil entre países y entre culturas parece una solución conceptual y metodológicamente muy débil ante la magnitud del reto planteado.

\footnotetext{
* Dirección para correspondencia [Correspondence address]:

Ferran Casas. Departamento Psicología. Universitat de Girona. Pl. S.

Domènec, 9.17071-Girona. E-mail: ferran.casas@udg.es
}

Title: Adolescents' subjective well-being: A comparative study between two Autonomous Communities in Spain.

Abstract: In two samples of 15 to 18 year-olds adolescents from Asturias and Catalonia inter-linguistic and intercultural comparability of subjective well-being is analysed. Three scales have been used as psychosocial indicators of well-being: The SWLS of Diener, Emmons, Larsen y Smith (1985), a context free scale, the PWI of Cummins, Eckersley, van Pallant, Vugt $y$ Misajon (2003), which evaluates personal well-being based on life domains, and a single-item scale on Overall Life Satisfaction (OLS).

Using Confirmatory Factor Analysis models, the PWI shows good fit for both samples, supporting the comparability of results between the two populations. Overall scores are higher among Asturian adolescents than among Catalan's, like OLS.

The SWLS shows bad fit when intercepts are restricted. That fact, added to that Cronbach alpha would increase if item 5 is deleted, gives support to the idea that this scale functions differently in diverse sociocultural contexts. These results raise doubts about its appropriateness as comparative instrument to be used with populations with different linguistic and/or sociocultural characteristics.

Key words: Subjective well-being; adolescence; PWI; SWLS; overall life satisfaction; personal well-being; confirmatory factor analysis; structural equation modelling.

Casas (2010) señala la disponibilidad de seis escalas para evaluar el bienestar subjetivo de población infantil no clínica, a partir de los ocho años, mientras que existen tres escalas de amplia utilización en población adulta que han sido utilizadas para población adolescente, mostrando un buen funcionamiento: El Personal Well-Being Index (PWI) (Cummins et al., 2003), la Satisfaction with Life Scale (SWLS) (Diener, Emmons, Larsen \& Smith, 1985) y la escala de ítem único de Fordyce (1988).

En poblaciones adultas, estas tres escalas han mostrado mantener correlaciones significativas, positivas y generalmente altas entre sí. Tienen características muy distintas, pero se considera que, en general, sus respectivos valores globales constituyen por igual buenos indicadores de bienestar subjetivo. Sin embargo, las correlaciones observadas no han sido suficientemente altas como para poder defender que miden constructos idénticos. Esta situación ha llevado a diversos autores a plantear la posible existencia de algún constructo de orden superior que abarque varios de los actualmente considerados a menudo sinónimos, medidos mediante escalas distintas (bienestar subjetivo, bienestar personal, satisfacción vital o felicidad, por ejemplo), con un aparente solapamiento entre ellos (Stones \& Kozma, 1985; Diener, Suh, Lucas \& Smith, 1999).

En un intento de aportar elementos novedosos a las lagunas existentes en los estudios sobre el bienestar de poblaciones adolescentes, en el presente artículo vamos a explorar el bienestar subjetivo de dos muestras de adolescentes de entre 15 y 18 años, de dos Comunidades Autónomas de carac- 
terísticas diferentes, Asturias y Cataluña (en la Tabla 1 pueden apreciarse algunos datos sociodemográficos distintivos de las dos comunidades autónomas). Los cuestionarios han sido administrados en idiomas distintos: en castellano en Asturias y en catalán en Cataluña.

Tabla 1. Algunos datos demográficos diferenciales entre Cataluña y Asturias.

\begin{tabular}{lccc}
\hline $\begin{array}{l}\text { Indicadores poblacionales } \\
(2008)\end{array}$ & Cataluña & Asturias & España \\
\hline Población general & $\begin{array}{c}7364078 \\
(15.95 \%)\end{array}$ & $\begin{array}{c}1080138 \\
(2.34 \%)\end{array}$ & $\begin{array}{c}46157882 \\
(100 \%)\end{array}$ \\
$\begin{array}{l}\text { Número de adolescentes } \\
(15-19 \text { años) }\end{array}$ & 341129 & 42700 & 2354504 \\
Número medio de hijos por & 1.57 & 1.08 & 1.45 \\
madre & & & \\
Número de inmigrantes & 923156 & 48058 & 4526522 \\
Crecimiento natural por 1000 & $(20 \%)$ & $(1 \%)$ & $(100 \%)$ \\
habitantes & 3.95 & -4.12 & 2.92 \\
\hline
\end{tabular}

Para ello hemos utilizado las dos escalas multi-item ampliamente conocidas (el PWI y la SWLS), cuyas versiones originales utilizaron escalas distintas: de 11 puntos el PWI (0-10) y de siete puntos la SWLS (1-7). No obstante, a partir de los planteamientos de Cummins \& Gullone (2000) que defienden la conveniencia de medir el bienestar subjetivo con escalas de más puntos, por su mayor sensibilidad, y dado el sesgo hacia el optimismo de las respuestas, algunos autores han incorporado escalas de 11 puntos al aplicar el SWLS (Casas et al., 2011) y este ha sido el caso de los datos de Cataluña del presente estudio.

Los objetivos son: (a) analizar el bienestar subjetivo de los adolescentes de dos poblaciones distintas; (b) comprobar el funcionamiento de dos instrumentos distintos para evaluar el bienestar subjetivo de los adolescentes, en contextos socio-culturales diferentes, (c) analizar el grado de compara- bilidad de los resultados entre las dos poblaciones y las posibles limitaciones que surjan.

\section{Método}

\section{Participantes}

Se obtuvo una muestra inicial de 2555 adolescentes, de la cual se eliminaron 16 que no respondieron ningún ítem del PWI (15 de Asturias y uno de Cataluña), más 17 que no respondieron ningún ítem de la SWLS (10 de Asturias y siete de Cataluña), más otros 13 que respondieron sólo dos ítems de una u otra escala. Así pues, la muestra final que se emplea en este artículo está compuesta por 2519 adolescentes de entre 15 y 18 años, matriculados en educación secundaria postobligatoria (Bachillerato o CFGM, es decir, Ciclo Formativo de Grado Medio, la antigua Formación Profesional reglada), de los cuales 1433 son de Cataluña y 1086 de Asturias. La distribución por edad y sexo puede observarse en la Tabla 2. En ella se observa una ligera mayor presencia de mujeres que refleja la realidad de esta población en ambas Comunidades Autónomas. En cuanto al menor número de los estudiantes de 18 años, se debe a que de esta edad generalmente sólo quedan alumnos que anteriormente han repetido algún curso.

Se ha utilizado un muestreo por conglomerados en dos etapas. En la primera se han seleccionado aleatoriamente algunos de los centros que tienen formación secundaria postobligatoria de la provincia de Girona y del Principado de Asturias. A continuación se informó a los directores de los centros y presidentes/as de las AMPAs sobre el proyecto, pidiendo su consentimiento. Entre los centros que aceptaron, se seleccionaron aleatoriamente una o dos aulas de cada nivel de estudios (dependiendo de si el centro tenía una línea o más) y se pidió la colaboración del profesor responsable.

Tabla 2. Distribución de la muestra $(\mathrm{N}=2.519)$ por territorio, edad y sexo.

\begin{tabular}{lcccccc}
\hline & \multicolumn{2}{c}{ Cataluña } & \multicolumn{2}{c}{ Asturias } & \multicolumn{2}{c}{ Total } \\
Edad & Chico & Chica & Chico & Chica & Chico & Chica \\
\hline 15 & 200 & 231 & 182 & 224 & 382 & 455 \\
16 & 245 & 365 & 237 & 254 & 482 & 619 \\
17 & 144 & 179 & 99 & 63 & 243 & 242 \\
18 & 26 & 43 & 20 & 7 & 46 & 50 \\
Total & 615 & 818 & 538 & 548 & 1153 & 1366 \\
\hline
\end{tabular}

\section{Instrumentos}

Personal Well-Being Index (PWI).- El PWI es una escala que fue diseñada como parte de la Australian Unit Wellbeing Index y cada uno de sus siete ítems originales evalúa, de forma relativamente genérica y abstracta, la satisfacción con un ámbito de la vida. Sus propiedades psicométricas han sido publicadas en varios artículos (Cummins et al., 2003; International Wellbeing Group, 2006; Lau, Cummins \& McPher- son, 2005). Sus valores van de "completamente insatisfecho" a "completamente satisfecho" con puntuaciones de 0 a 10 y solo llevan etiquetas los extremos. La escala global es el resultado de sumar los ítems y reconvertir el resultado a una escala de 0 a 100.

Aunque fue creada para su aplicación con adultos, ha sido probada con poblaciones a partir de 12 años en algunos países (Brasil, Chile, Rumania), mostrando buenas propiedades psicométricas (ver Casas et al., 2011). 
La escala original incluye un ítem sobre satisfacción con la comunidad que ha sido cambiado en varias de las adaptaciones para adolescentes a otras lenguas. En España las pruebas piloto mostraron que muchos adolescentes, particularmente los de entornos urbanos, no comprendían la pregunta. En el presente estudio, al igual que en estudios anteriores con muestras españolas, este ítem ha sido sustituido por uno de "satisfacción con los grupos a los que pertenezco" mostrando un buen funcionamiento con este cambio (Casas et al., 2011).

Satisfaction with Life Scale (SWLS).- La SWLS es definida por sus autores como una escala libre de contexto (context free), es decir, sus ítems son de carácter genérico y no se centran en ningún ámbito concreto de la vida. Consta de cinco ítems cuyos valores tienen todos una etiqueta que va desde "totalmente de acuerdo" a "totalmente en desacuerdo". Sus propiedades psicométricas han sido descritas en varias publicaciones, como por ejemplo en Pavot y Diener (1993) y en Diener et al. (1985). La escala original se responde mediante valores de 1 a 7 , aunque en la muestra catalana se ha utilizado con valores de 0 a 10 puntos para hacerla más sensible. En el presente estudio, los ítems de Asturias se han reconvertido aritméticamente a una escala 0 a 10 y el sumatorio de los ítems de cada sujeto se ha reconvertido, a su vez, en una escala de 0 a 100 para facilitar su comparación visualmente.

Existen varias adaptaciones al español de esta escala (ver, por ejemplo, Atienza, Pons, Balaguer y García-Merita, 2000; Castro y Sánchez-López, 2000), aunque ninguna de ellas realizada específicamente para adolescentes. Por esta razón, y también teniendo en cuenta que nuestros cuestionarios se administran en idiomas distintos en cada comunidad autónoma, hemos efectuado nuestra propia adaptación, garantizando la equivalencia en catalán y castellano, mediante triple comprobación por parte de investigadores bilingües.

Debido a su sentido negativo, la puntuación del ítem 5 se invierte para calcular la suma global de la escala. Dicho ítem ha sido criticado por varios autores, considerando que no mide bienestar subjetivo, sino actitudes ante nuevas experiencias (Veenhoven, 1994; 2009), por lo que se ha hipotetizado que puede ser problemático su uso con poblaciones adolescentes (Casas et al., 2011).

Tabla 3. Versiones inglesa original, y adaptadas para adolescentes al castellano y catalán, de las escalas utilizadas en este estudio.

\begin{tabular}{|c|c|c|c|}
\hline & Versión original en inglés & Versión adaptada al castellano & Versión adaptada al catalán \\
\hline PWI & $\begin{array}{l}\text { Thinking about your own life and } \\
\text { personal circumstances, how satis- } \\
\text { fied are you with ...? }\end{array}$ & $\begin{array}{l}\text { Actualmente, ¿hasta qué punto te } \\
\text { encuentras satisfecho o satisfecha } \\
\text { con cada una de estas cosas de tu } \\
\text { vida? }\end{array}$ & $\begin{array}{l}\text { Actualment, fins a quin punt et } \\
\text { trobes satisfet o satisfeta amb } \\
\text { cadascuna d'aquestes coses de la } \\
\text { teva vida? }\end{array}$ \\
\hline $\begin{array}{l}\text { PWI } \\
\text { Ítem } 1\end{array}$ & your health & Con tu salud & Amb la teva salut \\
\hline $\begin{array}{l}\text { PWI } \\
\text { Ítem } 2\end{array}$ & your standard of living & Con tu nivel de vida & Amb el teu nivell de vida \\
\hline $\begin{array}{l}\text { PWI } \\
\text { Ítem } 3\end{array}$ & your life achievements & $\begin{array}{l}\text { Con las cosas que has conseguido en la } \\
\text { vida }\end{array}$ & Amb les coses que has assolit a la vida \\
\hline $\begin{array}{l}\text { PWI } \\
\text { Ittem } 4\end{array}$ & your safety & Con lo seguro o segura que te sientes & Amb el segur o segura que et sents \\
\hline $\begin{array}{l}\text { PWI } \\
\text { Ítem } 5\end{array}$ & groups of people you belong to* & $\begin{array}{l}\text { Con los grupos de gente de los que formas } \\
\text { parte }\end{array}$ & $\begin{array}{l}\text { Amb els grups de gent dels quals form } \\
\text { part }\end{array}$ \\
\hline $\begin{array}{l}\text { PWI } \\
\text { Ittem } 6\end{array}$ & your future security & Con la seguridad por tu futuro & Amb la seguretat pel teu futur \\
\hline $\begin{array}{l}\text { PWI } \\
\text { Ítem } 7\end{array}$ & your interpersonal relationships & Con tus relaciones con las otras personas & $\begin{array}{l}\text { Amb les teves relacions amb les altres } \\
\text { persones }\end{array}$ \\
\hline OLS & your life as a whole & $\begin{array}{l}\text { Con toda tu vida, considerada } \\
\text { globalmente }\end{array}$ & $\begin{array}{l}\text { Amb tota la teva vida, considerada } \\
\text { globalment }\end{array}$ \\
\hline $\begin{array}{l}\text { SWLS } \\
\text { Ítem } 1\end{array}$ & $\begin{array}{l}\text { In most ways my life is close to my } \\
\text { ideal }\end{array}$ & $\begin{array}{l}\text { Mi vida, en casi todo, se } \\
\text { corresponde con aquello a lo que } \\
\text { aspiro }\end{array}$ & $\begin{array}{l}\text { La meva vida, en gairebé tot, es } \\
\text { correspon amb allò a que aspiro }\end{array}$ \\
\hline $\begin{array}{l}\text { SWLS } \\
\text { Ittem } 2\end{array}$ & $\begin{array}{l}\text { The conditions of my life are excel- } \\
\text { lent }\end{array}$ & $\begin{array}{l}\text { Las condiciones en que vivo son } \\
\text { buenas }\end{array}$ & $\begin{array}{l}\text { Les condicions en què visc són } \\
\text { bones }\end{array}$ \\
\hline $\begin{array}{l}\text { SWLS } \\
\text { Ítem } 3\end{array}$ & I am satisfied with my life & $\begin{array}{l}\text { Estoy satisfecho o satisfecha con } \\
\text { mi vida }\end{array}$ & $\begin{array}{l}\text { Estic satisfet o satisfeta amb la meva } \\
\text { vida }\end{array}$ \\
\hline $\begin{array}{l}\text { SWLS } \\
\text { Ítem } 4\end{array}$ & $\begin{array}{l}\text { So far I have gotten the important } \\
\text { things I want in life }\end{array}$ & $\begin{array}{l}\text { Hasta ahora, he conseguido cosas } \\
\text { que eran importantes para mí }\end{array}$ & $\begin{array}{l}\text { Fins ara, he aconseguit coses que } \\
\text { eren importants per a mi }\end{array}$ \\
\hline $\begin{array}{l}\text { SWLS } \\
\text { Ítem } 5\end{array}$ & $\begin{array}{l}\text { If I could live my life over, I would } \\
\text { change almost nothing }\end{array}$ & $\begin{array}{l}\text { Si volviese a nacer, cambiaría } \\
\text { bastantes cosas en mi vida }\end{array}$ & $\begin{array}{l}\text { Si tornés a néixer, canviaria bastants } \\
\text { coses en la meva vida }\end{array}$ \\
\hline
\end{tabular}


Ítem único de satisfacción global con la vida (Overall Life Satisfaction $=O L S)$.- La importancia de incluir en los estudios sobre bienestar subjetivo una escala de ítem único sobre satisfacción global con la vida fue subrayada ya por Campbell, Converse y Rodgers (1976). En esta investigación la incluimos con la formulación que se puede ver en la Tabla 3 , utilizando puntuaciones de 0 a 10 y con etiquetas únicamente en los valores extremos.

\section{Procedimiento}

Contando con la colaboración del profesorado, se pidió la participación voluntaria a todos los chicos y chicas de los grupos correspondientes, informándoles de que todas las respuestas serían tratadas anónima y confidencialmente. La administración se hizo grupalmente, generalmente en su propia aula, y con la presencia de uno de sus profesores y de uno o dos investigadores.

\section{Resultados}

\section{Análisis exploratorio}

Las respuestas medias obtenidas para cada escala y cada uno de sus ítems, según comunidad autónoma, se pueden observar en la Tabla 4.

Se aprecia a simple vista que las respuestas medias más bajas, con mucha diferencia, corresponden al ítem 5 de la SWLS. La más alta corresponde a la satisfacción con los grupos a los que se pertenece, seguida de la satisfacción con el nivel de vida y el ítem 2 de la SWLS, aunque este último ítem tiene un valor mucho más bajo en Asturias que en Cataluña.

De las tres escalas utilizadas, se observa que las puntuaciones globales medias son significativamente más elevadas en Asturias que en Cataluña tanto para el PWI $(F(1,2519)=19.39 ; \mathrm{p}=.000)$ como para la OLS $(F(1,2519)$ $=47.30 ; \mathrm{p}=.000)$, pero no así para la SWLS, cuyas diferencias entre muestras no alcanzan significación estadística $(F(1,2519)=2.80 ; \mathrm{p}=.094)$.

Las escalas muestran correlaciones relativamente altas entre sí, aunque notoriamente más altas en Asturias: ,549 entre PWI y SWLS (.483 en Cataluña y .634 en Asturias); .657 entre PWI y OLS (.628 en Cataluña y .689, en Asturias); y .498 entre SWLS y OLS (.432 en Cataluña y .612, en Asturias).

El $\alpha$ de Cronbach del PWI es .808 para la muestra global (.782 para la muestra catalana, y .839 para la asturiana), y ningún ítem incrementaría ese valor si fuera eliminado.

El $\alpha$ de Cronbach de la SWLS es .705 para la muestra global (.678, para la muestra catalana, y .744 para la asturiana), y se incrementaría de forma clara si el ítem 5 fuera eliminado: .733 (.721 en Cataluña; .760 en Asturias). Por tanto, aunque el ítem 5 es claramente un problema para la fiabilidad de la escala con la muestra catalana, su anula- ción también incrementaría el valor del $\alpha$ de Cronbach en la muestra asturiana.

Tabla 4. Resultados descriptivos de todos los ítems y escalas.

\begin{tabular}{|c|c|c|c|c|c|}
\hline & & & Cataluña & Asturias & Total \\
\hline \multirow{16}{*}{ PWI } & \multirow[t]{2}{*}{ Satisfacción salud } & $M$ & 7.94 & 8.25 & 8.07 \\
\hline & & $D T$ & 2.02 & 1.91 & 1.98 \\
\hline & \multirow{2}{*}{$\begin{array}{l}\text { Satisfacción nivel } \\
\text { vida }\end{array}$} & $M$ & 8.11 & 8.46 & 8.26 \\
\hline & & $D T$ & 1.69 & 1.63 & 1.67 \\
\hline & \multirow[t]{2}{*}{ Satisfacción logros } & $M$ & 7.54 & 7.61 & 7.57 \\
\hline & & $D T$ & 1.79 & 1.88 & 1.83 \\
\hline & \multirow{2}{*}{$\begin{array}{l}\text { Satisfacción seguro } \\
\text { sientes }\end{array}$} & $M$ & 7.15 & 7.20 & 7.17 \\
\hline & & $D T$ & 2.19 & 2.24 & 2.21 \\
\hline & \multirow{2}{*}{$\begin{array}{l}\text { Satisfacción grupos } \\
\text { pertenencia }\end{array}$} & $M$ & 8.33 & 8.49 & 8.40 \\
\hline & & $D T$ & 1.94 & 1.66 & 1.83 \\
\hline & \multirow{2}{*}{$\begin{array}{l}\text { Satisfacción segu- } \\
\text { ridad por futuro }\end{array}$} & $M$ & 6.80 & 7.30 & 7.01 \\
\hline & & $D T$ & 2.18 & 2.08 & 2.15 \\
\hline & \multirow{2}{*}{$\begin{array}{l}\text { Satisfacción rela- } \\
\text { ciones }\end{array}$} & $M$ & 7.98 & 8.17 & 8.06 \\
\hline & & $D T$ & 1.88 & 1.82 & 1.86 \\
\hline & \multirow[t]{2}{*}{ PWI TOTAL } & $\boldsymbol{M}$ & 76.92 & 79.25 & 77.93 \\
\hline & & $D T$ & 12.91 & 13.53 & 13.23 \\
\hline \multirow{12}{*}{$\begin{array}{l}\text { SW } \\
\text { LS }\end{array}$} & \multirow{2}{*}{$\begin{array}{l}\text { Vida corresponde } \\
\text { aspiro }\end{array}$} & $M$ & 6.22 & 6.55 & 6.36 \\
\hline & & $D T$ & 2.05 & 2.20 & 2.12 \\
\hline & \multirow{2}{*}{$\begin{array}{l}\text { Condiciones } \\
\text { buenas }\end{array}$} & $M$ & 8.57 & 7.47 & 8.10 \\
\hline & & $D T$ & 1.47 & 2.17 & 1.88 \\
\hline & \multirow[t]{2}{*}{ Satisfecho mi vida } & $M$ & 7.84 & 7.84 & 7.84 \\
\hline & & $D T$ & 1.92 & 2.08 & 1.99 \\
\hline & \multirow{2}{*}{$\begin{array}{l}\text { Conseguido } \\
\text { importantes }\end{array}$} & $M$ & 7.53 & 7.39 & 7.47 \\
\hline & & $D T$ & 1.95 & 2.24 & 2.08 \\
\hline & \multirow{2}{*}{$\begin{array}{l}\text { Cambiaría cosas mi } \\
\text { vida }\end{array}$} & $M$ & 5.05 & 5.48 & 5.23 \\
\hline & & $D$ & 3.31 & 3.51 & 3.40 \\
\hline & \multirow[t]{2}{*}{ SWLS TOTAL } & $\boldsymbol{M}$ & 70.41 & 69.47 & 70.01 \\
\hline & & $D T$ & 10.57 & 17.54 & 14.01 \\
\hline & \multirow[t]{2}{*}{ OLS } & & 7.81 & 8.31 & 8.03 \\
\hline & & $D T$ & 1.87 & 1.74 & 1.83 \\
\hline
\end{tabular}

Análisis factorial confirmatorio (AFC) y modelos de ecuaciones estructurales (MEE)

A fin de comprobar la validez de la estructura factorial de los datos de poblaciones de dos contextos socioculturales diferentes, se ha procedido primero a realizar pruebas con diferentes modelos de análisis factorial confirmatorio (AFC) de las dos escalas multi-ítem, utilizando el programa AMOS 19 para modelos de ecuaciones estructurales (MEE). Se ha utilizado la estimación de máxima verosimilitud. Los valores ausentes han sido imputados por regresión. El cálculo de los errores estándar se ha realizado mediante el método bootstrap dado que los datos muestran curtosis multivariada superior a la deseable. 
Como estadísticos de ajuste se han utilizado el CFI (Comparative Fix Index), el RMSEA (Root Mean Square Error of Approximation) y el SRMR (Standardized Root Mean Square Residual). Hemos asumido como aceptables los resultados del CFI por encima de .950 y los de los RMSEA y SRMR por debajo de .05 , tal y como recomiendan Batista-Foguet y Coenders (2000), Arbuckle (2010) y Byrne (2010). No obstante, también consideramos, como hacen algunos autores, que los valores de RMSEA hasta .08 representan errores de aproximación razonables en las muestras grandes (Browne \& Cudeck, 1993; Byrne, 2010).

Para poder comparar los coeficientes de los AFC entre grupos (en nuestro caso, entre comunidades autónomas) primero se debe comprobar que se da invarianza factorial. Se refiere al grado en que los ítems utilizados en un cuestionario significan lo mismo para los miembros de los distintos grupos estudiados y es un requisito para que la comparación de factores tenga sentido. En caso contrario, las diferencias de medias o de coeficientes de correlación podrían ser atribuidas a verdaderas diferencias en la distribución o a diferentes significados de las variables (Meredith, 1993). Consideraremos dos tipos de invarianza factorial: la llamada invarianza factorial métrica, que es un requisito para comparar varianzas, covarianzas o coeficientes de regresión entre grupos; y la denominada invarianza factorial fuerte, que es un requisito para poder comparar las medias de los factores entre grupos (Coenders, Batista-Foguer \& Saris, 2005).

Para ello desarrollaremos pruebas de ajuste estadístico de cada modelo en tres pasos. El primer paso consistirá en probar el ajuste de un modelo multigrupo sin ninguna restricción. El segundo paso será probar la invarianza factorial métrica mediante las cargas factoriales sin estandarizar restringidas entre grupos. Finalmente, probaremos la invarianza factorial fuerte restringiendo entre grupos los términos constantes de la ecuación.

\section{PWI}

Un AFC inicial, con las dos muestras agregadas, sin restricciones y sin permitir covariación de errores, relacionando los ítems del PWI a una variable latente, no mostró ajuste adecuado (Tabla 5, Modelo 1), como ya había sucedido con muestras catalanas anteriores, por lo que se pasó a poner a prueba el mismo modelo ajustado publicado en Casas et al. (2011), que incluye tres covarianzas de error: (a) satisfacción con las relaciones interpersonales y satisfacción con los grupos a los que se pertenece; (b) satisfacción con el nivel de vida y satisfacción con los propios logros; y (c) satisfacción con el nivel de vida y satisfacción con la salud.

Este Modelo modificado mostró un buen ajuste (Tabla 5, Modelo 2; en la Figura 1 se muestran las estimaciones estandarizadas), por lo que se pasó a poner a prueba como modelo multigrupo sin restricciones (Modelo 3), y a continuación el mismo modelo con cargas restringidas (Modelo 4) y con términos constantes restringidos (Modelo 5). No obstante, pudimos observar que el ajuste del Modelo 2 es muy distinto si se toma cada Comunidad Autónoma por separado, siendo el ajuste del RMSEA particularmente modesto en el caso de la muestra asturiana. El mayor índice de modificación observado es en Asturias (12.024), pero ninguno de los cambios predecibles estimados (Par Change) tiene sentido sustantivo (Byrne, 2010). Se observa una única discrepancia en la covariancia entre Satisfacción con los grupos a los que pertenezco y Satisfacción con mi nivel de vida, en Asturias(2.64), ligeramente superior al 2.58 que recomiendan Jöreskog \& Sörbom (1993).

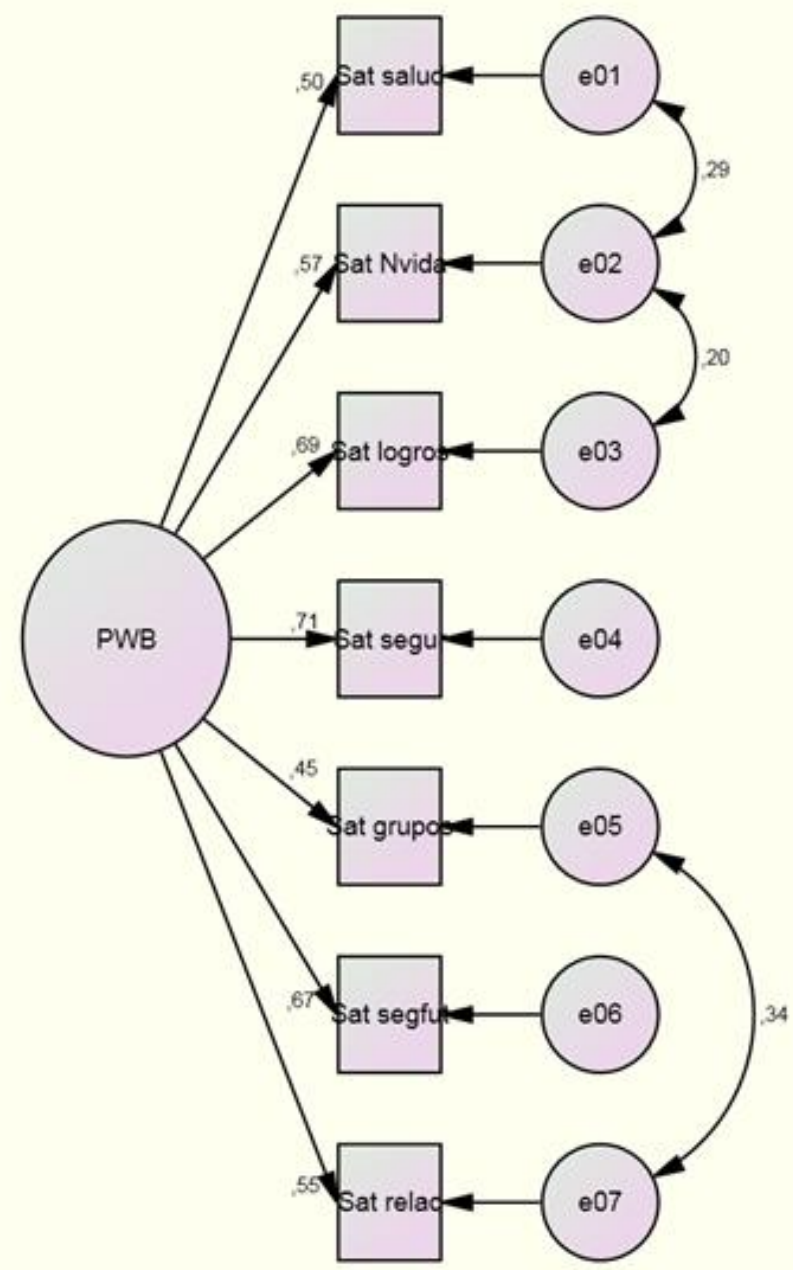

Figura 1. Análisis Factorial Confirmatorio del PWI: Pesos estandarizados con datos agregados de las dos CCAA. 
Dado que el Modelo 5 muestra un buen ajuste, se debe considerar que el índice compuesto con el que evalúa esta escala el bienestar personal resulta comparable entre las dos muestras estudiadas. En la Tabla 5 se presentan los índices de ajuste, y en la Tabla 6 las cargas factoriales estandarizadas del Modelo 5 con intervalos de confianza calculados con el método bootstrap.

Los datos de la Tabla 6 muestran que la satisfacción de los jóvenes asturianos es superior a la de los catalanes en cada uno de los siete ámbitos de la vida estudiados: la media del índice global del PWI difiere significativamente entre las dos muestras, la de Asturias superando a la de Cataluña en .223 puntos en una escala de 0 a 10 . También se aprecia que en las edades estudiadas los ámbitos de la vida que están más relacionados con la variable latente PWI de los adolescentes asturianos son los logros en la vida y la seguridad sentida, mientras que entre los adolescentes catalanes son los mismos, pero en orden inverso.

Aprovechando el buen funcionamiento de este modelo AFC, se ha puesto a prueba con la muestra global agregada un MEE completo, que incorpora la OLS, la edad y el sexo. El análisis inicial mostró que la covarianza de error entre la edad y el sexo no resulta significativa, por lo que no se ha incorporado al modelo. También se ha observado que el ajuste del modelo mejora añadiendo otra covarianza de error entre la satisfacción con la seguridad sentida y la satisfacción con la seguridad ante el futuro (Modelo 6). Al convertir este modelo en multigrupo se observa un buen ajuste, tanto sin restricciones, como con las cargas y las constantes restringidas (Modelo 7 de la Tabla 5). En la Tabla 7 se pueden consultar las estimaciones estandarizadas con intervalos de confianza calculados con el método bootstrap (ver también la Figura 2 para los datos de Asturias).

Tabla 5. Estadísticos de ajuste de los distintos modelos de ecuaciones estructurales analizados, utilizando el PWI.

\begin{tabular}{|c|c|c|c|c|c|c|c|c|}
\hline \multicolumn{2}{|c|}{ Modelo } & Muestra & $\chi^{2}$ & $\mathrm{gl}$ & $p$ & CFI & $\begin{array}{c}\text { RMSEA } \\
\text { (intervalo confianza) }\end{array}$ & SRMR \\
\hline 1 & Modelo inicial PWI & agregada & 568.41 & 14 & .000 & .887 & $\begin{array}{c}.125 \\
(.117-.134)\end{array}$ & .059 \\
\hline 2 & PWI modificado ( 3 errores covariando) & agregada & 68.28 & 11 & .000 & .988 & $\begin{array}{c}.045 \\
(.035-.056)\end{array}$ & .021 \\
\hline 3 & $\begin{array}{l}\text { PWI Modelo } 2 \\
\text { sin restricciones }\end{array}$ & multigrupo & 109.28 & 22 & .000 & .983 & $\begin{array}{c}.040 \\
(.032-.047)\end{array}$ & .017 \\
\hline 4 & $\begin{array}{l}\text { PWI Modelo } 2+ \\
\text { cargas restringidas }\end{array}$ & multigrupo & 134.33 & 28 & .000 & .979 & $\begin{array}{c}.039 \\
(.032-.046)\end{array}$ & .024 \\
\hline 5 & $\begin{array}{l}\text { PWI Modelo 2+cargas + constantes } \\
\text { restringidas }\end{array}$ & multigrupo & 188.34 & 34 & .000 & .969 & $\begin{array}{c}.042 \\
(.037-.048)\end{array}$ & .023 \\
\hline 6 & $\begin{array}{l}\text { PWI Modelo } 2+\text { OLS }+ \text { edad }+ \text { sexo } \\
(4 \text { errores covariando })\end{array}$ & agregada & 222.63 & 28 & .000 & .970 & $\begin{array}{c}.053 \\
(.046-.059)\end{array}$ & .029 \\
\hline 7 & $\begin{array}{l}\text { PWI Modelo } 6+\text { cargas + constantes } \\
\text { restringidas }\end{array}$ & multigrupo & 356.34 & 68 & .000 & .956 & $\begin{array}{c}.041 \\
(.037-.045)\end{array}$ & .033 \\
\hline
\end{tabular}

Nota: El Modelo 2 muestra ajustes notoriamente distintos si se toma cada Comunidad Autónoma por separado. Cataluña: $\chi^{2}=25.45$; $\mathrm{gl}=11 ; \mathrm{p}=.008 ; \mathrm{CFI}=.994 ; \mathrm{RMSEA}=.030(.015-.046) ; \mathrm{SRMR}=.017$. Asturias: $\chi^{2}=83.83 ; \mathrm{gl}=11 ; \mathrm{p}=.000 ; \mathrm{CFI}=.973 ; \mathrm{RMSEA}=.078(.063-$ $.094) ; \mathrm{SRMR}=.034$.

Tabla 6. Análisis Factorial Confirmatorio del PWI mediante modelo multigrupo, con cargas y constantes restringidas. Cargas factoriales estandarizadas y diferencia de medias de factor (Modelo 5).

\begin{tabular}{|c|c|c|c|c|c|c|c|c|}
\hline \multirow{2}{*}{\multicolumn{3}{|c|}{$\begin{array}{l}\text { Bootstrap ML. al } 95 \% \text { de confianza. } \\
\text { Muestras }=500\end{array}$}} & \multicolumn{3}{|c|}{ Cataluña } & \multicolumn{3}{|c|}{ Asturias } \\
\hline & & & \multirow{2}{*}{$\begin{array}{c}\text { Estim } \\
.467\end{array}$} & \multirow{2}{*}{$\frac{\text { Inferior }}{.423}$} & \multirow{2}{*}{$\frac{\text { Superior }}{.514}$} & \multirow{2}{*}{$\begin{array}{c}\text { Estim } \\
.558\end{array}$} & \multirow{2}{*}{$\frac{\text { Inferior }}{.505}$} & \multirow{2}{*}{$\frac{\text { Superior }}{.616}$} \\
\hline Sat salud & $<---$ & PWI & & & & & & \\
\hline Sat nivel vida & $<---$ & PWI & .543 & .494 & .589 & .629 & .568 & .684 \\
\hline Sat logros en la vida & $<---$ & PWI & .654 & .608 & .691 & .751 & .702 & .786 \\
\hline Sat seguridad sentida & $<---$ & PWI & .674 & .632 & .711 & .743 & .703 & .775 \\
\hline Sat grupos pertenencia & $<---$ & PWI & .400 & .353 & .451 & .509 & .453 & .576 \\
\hline Sat seguridad futuro & $<---$ & PWI & .621 & .577 & .666 & .717 & .671 & .755 \\
\hline Sat relaciones interp & $<---$ & PWI & .517 & .462 & .572 & .586 & .528 & .647 \\
\hline PWI (Medias, no estand.) & & & $0^{1}$ & 0 & 0 & .223 & .120 & .339 \\
\hline
\end{tabular}

\footnotetext{
${ }^{1}$ Cataluña se usa como nivel de referencia en la comparación de medias del PWI.
} 
Tabla 7. MEE multigrupo relacionando la OLS, la edad y el sexo, con el PWI; cargas y constantes restringidas. Estimaciones estandarizadas (Modelo 7).

\begin{tabular}{|c|c|c|c|c|c|c|c|c|}
\hline \multirow{2}{*}{\multicolumn{2}{|c|}{$\begin{array}{l}\text { Bootstrap ML. al 95\% de confianza. } \\
\text { Muestras }=500\end{array}$}} & & \multicolumn{3}{|c|}{ Cataluña } & \multicolumn{3}{|c|}{ Asturias } \\
\hline & & & Estim & Inferior & Superior & Estim & Inferior & Superior \\
\hline OLS & $<---$ & Edad & $-.021 *$ & -.070 & .033 & $-.028 *$ & -.093 & .041 \\
\hline OLS & $<---$ & Sexo & $-.054 *$ & -.106 & .003 & $-.056 *$ & -.117 & .012 \\
\hline (PWI) & $<---$ & OLS & .736 & .679 & .780 & .755 & .705 & .801 \\
\hline (PWI) & $<---$ & Edad & -.081 & -.132 & -.025 & $-.032 *$ & -.085 & .024 \\
\hline (PWI) & $<---$ & Sexo & $-.029 *$ & -.081 & .017 & -.063 & -.114 & -.013 \\
\hline Sat salud & $<---$ & PWI & .492 & .446 & .537 & .590 & .537 & .651 \\
\hline Sat nivel vida & $<---$ & PWI & .598 & .547 & .644 & .674 & .617 & .724 \\
\hline Sat logros en la vida & $<---$ & PWI & .663 & .622 & .700 & .748 & .698 & .788 \\
\hline Sat seguridad sentida & $<---$ & PWI & .626 & .581 & .665 & .706 & .651 & .745 \\
\hline Sat grupos pertenencia & $<---$ & PWI & .406 & .357 & .458 & .521 & .463 & .579 \\
\hline Sat seguridad futuro & $<---$ & PWI & .574 & .528 & .619 & .683 & .640 & .721 \\
\hline Sat relaciones interperson. & $<---$ & PWI & .518 & .465 & .570 & .595 & .541 & .654 \\
\hline
\end{tabular}

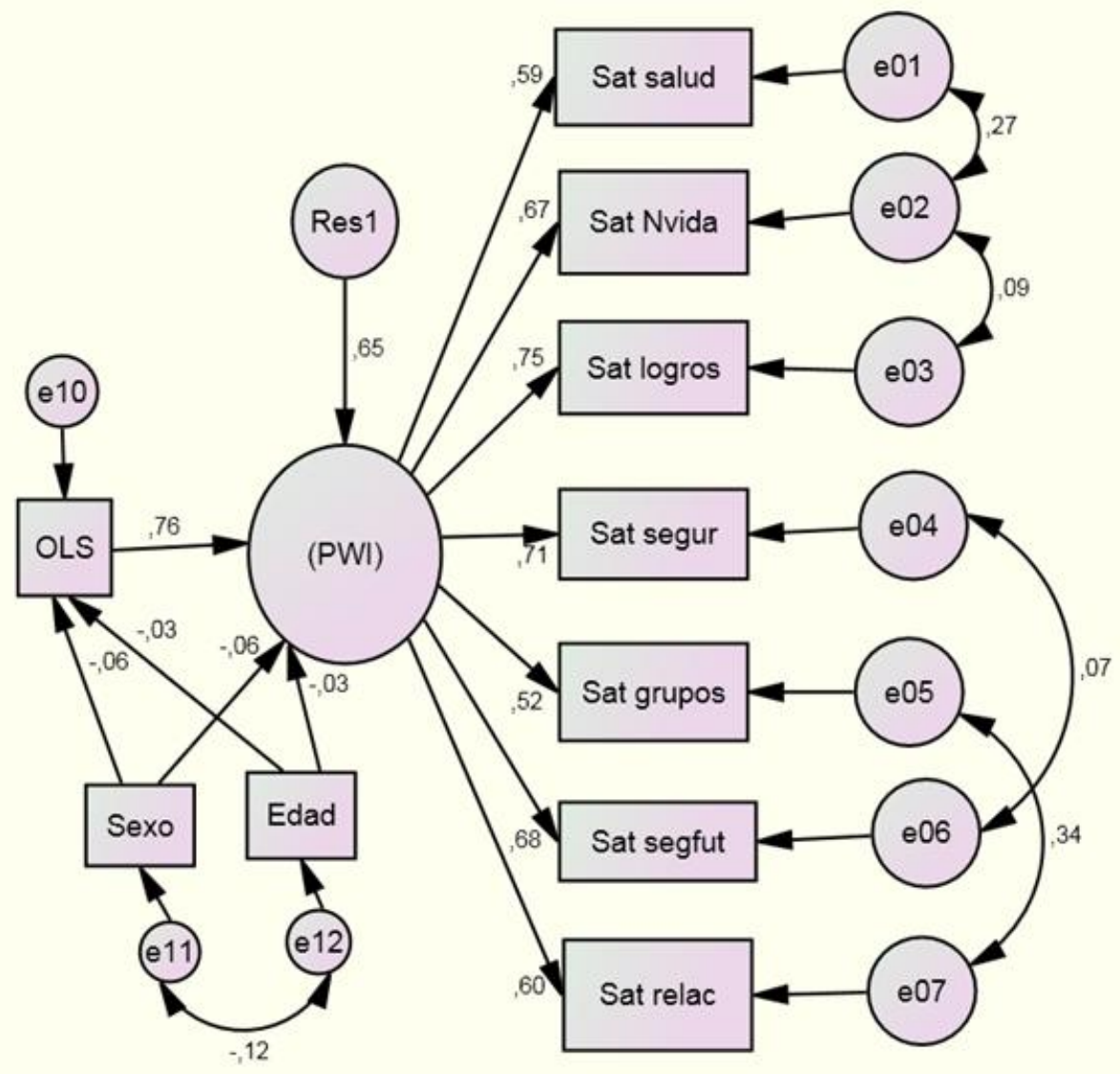

Figura 2. MEE multigrupo que relaciona el PWI con el OLS, edad y sexo. Pesos estandarizados de Asturias (Modelo 7).

Esta Tabla nos muestra que, durante el intervalo de edad estudiado, el PWI tiene una relación inversa con la edad, aunque baja y sólo para la muestra catalana, mientras que el sexo la mantiene sólo para la muestra asturiana, siendo los chicos quienes presentan puntuaciones más altas. La OLS no alcanza significación estadística en ninguna de las dos muestras ni con el sexo ni con la edad. 


\section{SWLS}

Un AFC inicial sin restricciones y sin permitir covariación de errores, relacionando los ítems de la SWLS a una variable latente, mostró un buen ajuste estadístico (Modelo 8 de la Tabla 8). En la Figura 3 se muestran las cargas factoria- les estandarizadas. No obstante, al igual que ocurrió con el PWI, pudimos observar que el ajuste del Modelo 8 es muy distinto si se toma cada Comunidad Autónoma por separado, siendo el ajuste del RMSEA particularmente malo en el caso de la muestra asturiana.

Tabla 8. Estadísticos de ajuste de los distintos modelos de ecuaciones estructurales analizados, utilizando la SWLS.

\begin{tabular}{|c|c|c|c|c|c|c|c|c|}
\hline \multicolumn{2}{|c|}{ Modelo } & \multirow{2}{*}{$\begin{array}{l}\text { Muestra } \\
\text { agregada }\end{array}$} & \multirow{2}{*}{$\begin{array}{c}\chi^{2} \\
30.45\end{array}$} & \multirow{2}{*}{$\begin{array}{r}\text { gl } \\
5\end{array}$} & \multirow{2}{*}{$\begin{array}{c}\mathrm{p} \\
.000\end{array}$} & \multirow{2}{*}{$\begin{array}{r}\text { CFI } \\
.990\end{array}$} & \multirow{2}{*}{$\begin{array}{c}\text { RMSEA } \\
\text { (intervalo confianza) } \\
.045 \\
(.030-.061)\end{array}$} & \multirow{2}{*}{$\begin{array}{c}\text { SRMR } \\
.018\end{array}$} \\
\hline 8 & Modelo inicial SWLS & & & & & & & \\
\hline 9 & $\begin{array}{l}\text { SWLS Modelo } 8 \\
\text { sin restricciones }\end{array}$ & multirupo & 57.41 & 10 & .000 & .983 & $\begin{array}{c}.043 \\
(.033-.055)\end{array}$ & .016 \\
\hline 10 & $\begin{array}{l}\text { SWLS Modelo } 8+ \\
\text { cargas restringidas }\end{array}$ & multigrupo & 134.59 & 14 & .000 & .957 & $\begin{array}{c}.058 \\
(.050-.068)\end{array}$ & .031 \\
\hline 11 & $\begin{array}{l}\text { SWLS Modelo } 8 \\
\text { + cargas+constantes restringidas }\end{array}$ & multigrupo & 470.26 & 18 & .000 & .839 & $\begin{array}{c}.100 \\
(.092-.108)\end{array}$ & .027 \\
\hline 12 & $\begin{array}{l}\text { SWLS Modelo } 10+\text { OLS + } \\
\text { edad }+ \text { sexo }\end{array}$ & multigrupo & 239.00 & 38 & .000 & .953 & $\begin{array}{c}.046 \\
(.040-.051)\end{array}$ & .032 \\
\hline
\end{tabular}

Nota: El Modelo 8 muestra ajustes notoriamente distintos si se toma cada Comunidad Autónoma por separado. Cataluña: $\chi 2=13.05$; gl=5; $\mathrm{p}=.023$; CFI= $.994 ;$ RMSEA=.034(.011-.056); SRMR=.016. Asturias: $\chi 2=44.36 ; \mathrm{gl}=5 ; \mathrm{p}=.000 ; \mathrm{CFI}=.972 ; \mathrm{RMSEA}=.085(.063-.109) ; \mathrm{SRMR}=.029$.

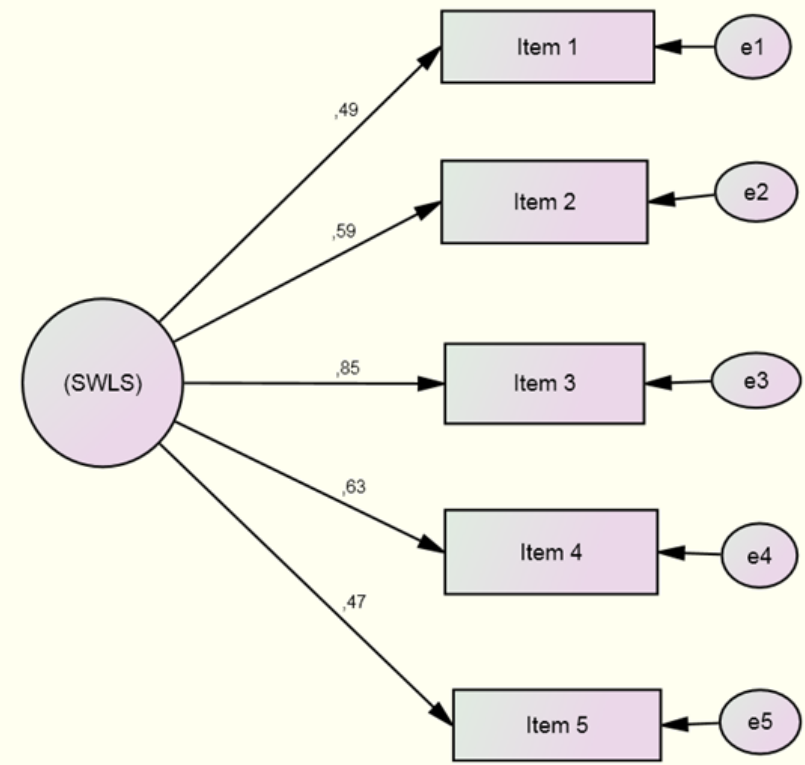

Figura 3. Análisis Factorial Confirmatorio de la SWLS: Pesos estandarizados con datos agregados de las dos CCAA.

Pasamos a poner a prueba este modelo como multigrupo sin restricciones (Modelo 9), y a continuación con cargas restringidas (Modelo 10) y con términos constantes restringidos (Modelo 11). Los Modelos 9 y 10 muestran un buen ajuste, mientras que el Modelo 11 no (Tabla 8), indicando que el índice compuesto con el que esta escala evalúa el bienestar personal no resulta comparable entre las dos muestras estudiadas. Pueden verse las cargas factoriales estandarizadas calculadas con el Modelo 10 para ambas muestras en la Tabla 9. El análisis muestra que en ambas muestras el ítem 5 es el que presenta la menor contribución a la variable latente SWLS, mientras que el ítem 3 muestra una carga muy superior a todos los demás.

A partir del Modelo 8, pusimos a prueba otro MEE multigrupo, que además de la variable latente SWLS, incluía la OLS, la edad y el sexo, y que mostró un buen ajuste. Tal como era de esperar, al poner a prueba este nuevo modelo sin restricciones, y con cargas restringidas (Modelo 12), en ambos casos mostró un buen ajuste, pero no así cuando se restringieron los términos constantes. En la Tabla 10 pueden observarse los coeficientes estandarizados de cada muestra por separado, con cargas factoriales restringidas, que indican que la SWLS muestra una relación significativa con la edad en Asturias, pero no en Cataluña, mientras que su relación con el sexo no alcanza la significación en ninguna de las dos muestras (ver los de Cataluña en la Figura 4). La contribución de la OLS sobre la SWLS resulta mayor en la muestra catalana que en la asturiana. Sólo el ítem 2 muestra una contribución a la variable latente SWLS superior en la muestra catalana que en la asturiana. 
Tabla 9. Análisis Factorial Confirmatorio de la SWLS mediante modelo multigrupo; cargas restringidas. Cargas factoriales estandarizadas (Modelo 10).

\begin{tabular}{|c|c|c|c|c|c|c|c|c|}
\hline \multirow{2}{*}{\multicolumn{3}{|c|}{$\begin{array}{l}\text { Bootstrap ML. al 95\% de confianza. } \\
\text { Muestras }=500\end{array}$}} & \multicolumn{3}{|c|}{ Cataluña } & \multicolumn{3}{|c|}{ Asturias } \\
\hline & & & \multirow{2}{*}{$\frac{\text { Estim }}{.468}$} & \multirow{2}{*}{$\frac{\text { Inferior }}{.416}$} & \multirow{2}{*}{$\frac{\text { Superior }}{.518}$} & \multirow{2}{*}{$\frac{\text { Estim }}{.505}$} & \multirow{2}{*}{$\frac{\text { Inferior }}{.448}$} & \multirow{2}{*}{$\frac{\text { Superior }}{.560}$} \\
\hline SWLS1 & $<---$ & (SWLS) & & & & & & \\
\hline SWLS2 & $<---$ & (SWLS) & .611 & .567 & .651 & .525 & .460 & .589 \\
\hline SWLS3 & $<---$ & (SWLS) & .822 & .771 & .866 & .869 & .835 & .904 \\
\hline SWLS4 & $<---$ & (SWLS) & .629 & .587 & .671 & .630 & .582 & .677 \\
\hline SWLS5 & $<---$ & (SWLS) & .444 & .403 & .483 & .501 & .456 & .544 \\
\hline
\end{tabular}

Tabla 10. MEE multigrupo relacionando la OLS, la edad y el sexo, con la SWLS; cargas restringidas. Estimaciones estandarizadas (Modelo 12).

\begin{tabular}{|c|c|c|c|c|c|c|c|c|}
\hline \multirow{2}{*}{\multicolumn{3}{|c|}{$\begin{array}{l}\text { Bootstrap ML. al 95\% de confianza. } \\
\text { Muestras }=500\end{array}$}} & \multicolumn{3}{|c|}{ Cataluña } & \multicolumn{3}{|c|}{ Asturias } \\
\hline & & & \multirow{2}{*}{$\frac{\text { Estim }}{-.021 *}$} & \multirow{2}{*}{$\frac{\text { Inferior }}{-.070}$} & \multirow{2}{*}{$\frac{\text { Superior }}{.033}$} & \multirow{2}{*}{$\frac{\text { Estim }}{-.028 *}$} & \multirow{2}{*}{$\frac{\text { Inferior }}{-.093}$} & \multirow{2}{*}{$\frac{\text { Superior }}{.041}$} \\
\hline$\overline{\mathrm{OLS}}$ & $<---$ & Edad & & & & & & \\
\hline OLS & $<---$ & Sexo & $-.054 *$ & -.106 & .003 & $-.056 *$ & -.117 & .012 \\
\hline (SWLS) & $<---$ & OLS & .750 & .701 & .789 & .692 & .632 & .745 \\
\hline (SWLS) & $<---$ & Edad & $-.018 *$ & -.057 & .020 & -.045 & -.080 & -.008 \\
\hline (SWLS) & $<---$ & Sexo & $-.008 *$ & -.055 & .039 & $-.021 *$ & -.066 & .031 \\
\hline SWLS1 & $<---$ & (SWLS) & .475 & .428 & .520 & .507 & .451 & .558 \\
\hline SWLS2 & $<---$ & (SWLS) & .596 & .555 & .630 & .505 & .442 & .562 \\
\hline SWLS3 & $<---$ & (SWLS) & .842 & .797 & .877 & .852 & .818 & .883 \\
\hline SWLS4 & $<---$ & (SWLS) & .629 & .589 & .668 & .632 & .588 & .675 \\
\hline SWLS5 & $<---$ & (SWLS) & .458 & .421 & .495 & .510 & .465 & .555 \\
\hline
\end{tabular}

* No alcanza significación estadística

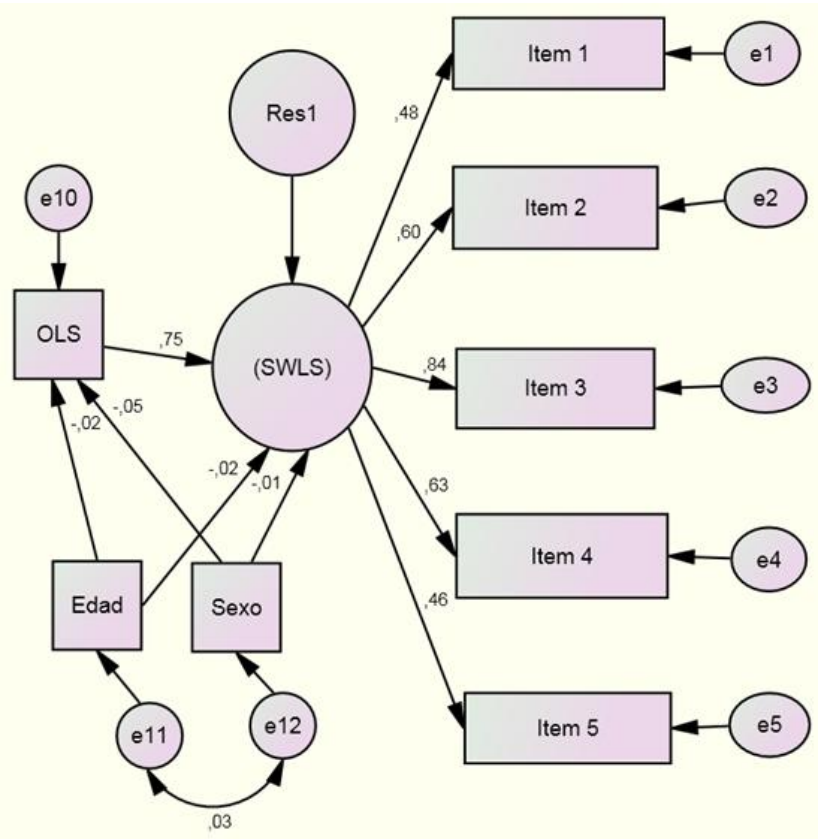

Figura 4. MEE multigrupo que relaciona el SWLS con el OLS, edad y sexo. Pesos estandarizados de Cataluña (Modelo 12).

\section{Discusión}

Los adolescentes entre 15 y 18 años de las dos comunidades autónomas estudiadas muestran unos niveles de bienestar subjetivo muy alto, evaluado con cualquiera de las tres escalas utilizadas (PWI, SWLS, OLS), aunque los valores medios de la SWLS son comparativamente inferiores de forma clara a los de las otras dos escalas. Los resultados de todos los ítems estudiados son siempre notoriamente más altos para Asturias que para Cataluña, excepto en el caso del ítem 2 de la SWLS. En el caso del PWI los resultados de Asturias (M $=79.25)$ rozan el límite superior normativo esperado para poblaciones de los países occidentales que, según Cummins y Cahil (2000), estaría en 80 puntos sobre 100, y mediante un AFC multigrupo comparando ambos índices, se observa que la media de la muestra asturiana resulta significativamente superior a la de la catalana.

Los AFC de cada una de las dos escalas multi-ítem muestran un buen ajuste de un Modelo modificado del PWI y del Modelo inicial de la SWLS. Sin embargo, el análisis desagregado por Comunidades Autónomas nos ha mostrado un RMSEA modesto para el PWI y malo para la SWLS en la muestra asturiana. Un modelo multigrupo de la SWLS con cargas y constantes restringidas no ajusta, demostrando que, ni los índices de la escala, ni sus medias son comparables entre ambas muestras. No obstante, con sólo las cargas restringidas, el modelo presenta un buen ajuste, a efectos de relacionar la SWLS con variables externas. 
Aunque el hecho de haber administrado la SWLS con distintas escalas numéricas en las dos muestras (de siete puntos en la asturiana y de 11 en la catalana) por si solo podría ocasionar la falta de ajuste del modelo multigrupo, la observación de que el modelo de la muestra asturiana por separado no ajuste parece apoyar más bien la posición de aquellos autores que defienden el uso de escalas con mayores rangos de puntuación, por su mayor sensibilidad y capacidad discriminativa. No obstante, no podemos descartar la posibilidad que los resultados aquí ofrecidos sean debidos simplemente a un diferente estilo de respuesta de los adolescentes asturianos ante la misma escala, lo cual deberá ser objeto de ulteriores investigaciones.

Los resultados también abundan en la duda de si SWLS es una escala adecuada para utilizar con adolescentes dado que, por una parte la supresión del ítem 5 aumentaría el valor de la alpha de Cronbach en ambas muestras, y por otra, las medias de cada unos de sus 5 ítems se muestran notablemente dispares en ambas muestras (Tabla 4). Finalmente, el ítem 3 presenta unas cargas factoriales estandarizadas muy superiores a los demás ítems en ambas muestras, sugiriendo que la escala está descompensada, tal y como ya se ha apuntado en otras investigaciones (Casas et al., 2011).

En contraste, el PWI muestra un buen ajuste en un Modelo multigrupo con cargas y constantes restringidas, permitiendo así la comparación entre muestras. En dicha comparación se confirma la superior puntuación de los jóvenes asturianos en cada uno de los ámbitos del bienestar personal y en el índice global. Entre ambas muestras sólo se observa una diferencia en el orden de los dos ámbitos con mayor peso: en Asturias los ámbitos de la vida que más contribuyen al bienestar personal son los logros en la vida y la seguridad sentida, mientras que en Cataluña son los mismos, pero en orden inverso. No obstante, al introducir la OLS, la edad y el sexo en el Modelo, también en la muestra catalana los logros en la vida pasan a ser el ámbito de mayor peso (Tabla 7).

Tanto los resultados de las tres escalas, como buena parte de los ítems, muestran una cierta tendencia a la baja con la edad, aunque algunos ítems parecen recuperarse a los 18 años en la muestra de Asturias. Probablemente este resultado sugiere que el claro descenso del bienestar subjetivo observado en diversas poblaciones entre los 12 y los 16 años se modera al estudiarlo en poblaciones de mayor edad, como es nuestro caso (15-18 años), difiriendo en muestras de distintos contextos y mostrando resultados distintos según el instrumento utilizado. Los modelos de ecuaciones estructurales (MEE) con las variables edad, sexo y la OLS, con las respectivas variables latentes aquí estudiadas, muestran una correlación inversa entre la edad y el PWI sólo en la muestra cata-

\section{Referencias}

Adamson, P. (Ed.) (2007). Child Poverty in perspective: An overview of child wellbeing in rich countries. Report Card 7. Innocenti Research Centre. UNICEF. Arbuckle, J.L. (2010). IBM SPSS® Amos ${ }^{\mathrm{TM}} 19$ User's Guide. Crawfordville (Fl): Amos Development Corporation. lana, y con la edad y la SWLS sólo en la asturiana, no alcanzando significación estadística con la OLS en ninguna de las dos muestras.

Hemos visto que las tres escalas aquí utilizadas mantienen correlaciones relativamente altas entre si y que la correlación más alta es entre PWI y OLS en ambas muestras. Al incorporar la OLS a los respectivos modelos con las variables latentes de los ítems de las otras dos escalas, observamos que, respectivamente, los coeficientes estandarizados de la OLS sobre el PWI son de .736 en la muestra catalana y .755 en la asturiana, mientras que los de la OLS sobre la SWLS son .750 para la catalana y .692 para la asturiana.

Aunque todos estos resultados muestran que la SWLS es una escala que funciona aceptablemente, muchos de los aspectos aquí expuestos parecen sugerir que el PWI es una escala con cualidades más adecuadas para estudios comparativos entre poblaciones adolescentes de diferentes entornos lingüísticos y socio-culturales. El PWI muestra unas buenas propiedades psicométricas y su alta correlación tanto con la OLS como con la SWLS pueden considerase indicativas de validez concurrente. El modelo modificado aquí estudiado, permitiendo algunas covariaciones de errores entre ítems, muestra un buen ajuste estadístico.

El presente estudio muestra una vez más que, en la evaluación del bienestar subjetivo, la elección del instrumento no es un asunto banal, como tampoco lo es el rango de puntuaciones que utiliza cada instrumento. A pesar de que los tres instrumentos para la medición del bienestar subjetivo acostumbran a mantener correlaciones relativamente altas entre ellos, como también ha sucedido en el presente estudio, vemos que los resultados que ofrecen para cada muestra tienen características distintas.

Dado que el presente estudio se ha limitado a muestras por conglomerados de sólo dos comunidades autónomas, en el futuro sería interesante recoger datos de muestras por conglomerados más grandes, estratificando algunas variables, como por ejemplo el hecho de provenir de medio rural, semiurbano o urbano, y de más comunidades autónomas, ampliando el número de escalas utilizadas, y adaptando todos los instrumentos para ser administrados con escalas de 0 a 10 puntos, a fin de poder profundizar en la comparabilidad de los resultados entre poblaciones de distintas características y en la adecuación para este fin de los distintos instrumentos disponibles.

Reconocimientos: La recogida de datos de Cataluña ha contado con la ayuda económica del Ministerio de Educación y Ciencia, referencia SEJ2007-62813/PS.

Atienza, F.L., Pons, D., Balaguer, I., y García-Merita, M. (2000). Propiedades psicométricas de la Escala de Satisfacción con la Vida en adolescentes. Psicothema, 12(2), 314-319.

Batista-Foguet, J. M. y Coenders, G. (2000). Modelos de ecuaciones estructurales. Madrid: La Muralla. 
Browne, M.W., y Cudeck, R. (1993). Alternative ways of assessing model fit. En K.A. Bollen y J.S. Long (eds.), Testing structural equation models (pp. 136162). Thousand Oaks: Sage.

Byrne, B. M. (2010). Structural Equation Modeling with AMOS. Basic concepts, Applications and Programming. New York: Routledge.

Campbell, A., Converse, P.E., y Rodgers, W.L. (1976). The quality of American life: Perceptions, evaluations, and satisfactions. Nueva York: Russell Sage.

Cantril, H. (1965). The pattern of human concerns. New Brunswick, N.J., Rutgers Univ. Press.

Casas, F. (2010). El bienestar personal: Su investigación en la infancia y la adolescencia. Encuentros en Psicología, 5(1), 85-101.

Casas, F., Sarriera, J.C., Abs, D., Coenders, G., Alfaro, J., Saforcada, E. y Tonon, G. (2011). Subjective indicators of personal well-being among adolescents. Performance and results for different scales in Latinlanguage speaking countries: A contribution to the international debate. Child Indicators Research. Accepted.

Castro, A., y Sánchez-López, M.P. (2000). Objetivos de vida y satisfacción autopercibida en estudiantes universitarios. Psicothema, 12 (1), 87-92.

Coenders, G. y Batista-Foguet, J.M. (2005). Modelos de ecuaciones estructurales. Madrid: La Muralla.

Coenders, G., Batista-Foguet, J.M. y Saris, W. (2005). Temas avanzados en modelos de ecuaciones estructurales. Madrid: La Muralla.

Cummins, R.A. y Cahill, J. (2000). Avances en la comprensión de la calidad de vida subjetiva. Intervención Psicosocial, 9 (2), 185-198.

Cummins, R.A., Eckersley, R., Lo, S.K., Okerstrom, E., Hunter, B., y Davern, M. (2003). Australian Unity Wellbeing Index: Cumulative Psychometric Record. Report 9.0. Melbourne: Australian Centre on Quality of Life. http://www.deakin.edu.au/research/acqol/instruments/PWI/Cumulativ e Psychometric Record Australian data.doc

Cummins, R.A., Eckersley, R., van Pallant, J., Vugt, J. y Misajon, R. (2003). Developing a national index of subjective well-being: The Australian Unity Well-being Index. Social Indicators Research, 64, 159-190. (Updated in: http://www.deakin.edu.au/research/acqol/instruments/wellbeing index htm
Cummins, R.A. y Gullone, E. (2000) Why we should not use 5-point Likert scales: The case for subjective quality of life measurement. Proceedings Second International Conference on Quality of Life in Cities (pp. 74-93). Singapore: National University of Singapore.

Diener, E., Emmons, R., Larsen, R., y Smith, H.L. (1985). The satisfaction with life scale. Journal of Personality Assesment, 49(1), 71-75.

Diener, E., Suh, E.M., Lucas, R.E. y Smith, H.L. (1999). Subjective wellbeing: Three decades of progress. Psychological Bulletin, 125(2), 276-302.

Fordyce, M.W. (1988). A review of research on the happiness measures: a sixty second index of happiness and mental health. Social Indicators Research, 20(4), 355-381.

Huebner, E.S. (2004). Research on assessment of life satisfaction of children and adolescents. Social Indicators Research, 66(1-2), 3-33.

International Wellbeing Group (2006). Personal Wellbeing Index - Adult - Manual, 4th version. Melbourne: Australian Centre on Quality of Life, Deakin University. http://www.deakin.edu.au/research/acqol/instruments/wellbeing index.htm

Jöreskog, K. G., \& Sörbom, D. (1993). LISREL 8: Structural equation modeling with the SIMPLIS command language. Chicago: Scientific Software International.

Lau, A.L.D., Cummins, R.A., y McPherson, W. (2005). An investigation into the cross-cultural equivalence of the Personal Well-Being Index. Social Indicators Research, 72, 403-430.

Meredith, W. (1993). Measurement invariance, factor analysis and factorial invariance, Psychometrika, 58, 525-543.

Pavot, W., y Diener, E. (1993). Review of the Satisfaction with Life Scale. Psychological Assessment, 5(2), 164-172.

Stones, M.J., y Kozma, A. (1985). Structural relationships among happiness scales: A second order factorial study. Social Indicators Research, 17, 19-28.

Veenhoven, R. (1994). El estudio de la satisfacción con la vida. Intervención Psicosocial, III (9), 87-116; \& IV (10), 125-127.

Veenhoven, R. (2009). Medidas de felicidad nacional bruta. Intervención Psicosocial, 18(3), 279-299.

(Articulo recibido: 27-01-2012, revisado: 17-03-2012, aceptado: 17-03-2012) 